\title{
Structure of the Interstellar Medium Around Cas A
}

\author{
Yeunjin Kim ${ }^{1,2}$, G. H. Rieke ${ }^{1}$, O. Krause $^{3}$, K. Misselt ${ }^{1}$, R. Indebetouw ${ }^{4}$, K. E. Johnson ${ }^{4}$
}

\begin{abstract}
We present a three-year series of observations at $24 \mu \mathrm{m}$ with the Spitzer Space Telescope of the interstellar material in a 200 x 200 arcmin square area centered on Cassiopeia A. Interstellar dust heated by the outward light pulse from the supernova explosion emits in the form of compact, moving features. Their sequential outward movements allow us to study the complicated three-dimensional structure of the interstellar medium (ISM) behind and near Cassiopeia A. The ISM consists of sheets and filaments, with many structures on a scale of a parsec or less. The spatial power spectrum of the ISM appears to be similar to that of fractals with a spectral index of 3.5. The filling factor for the small structures above the spatial wavenumber $k \sim 0.5$ cycles $\mathrm{pc}^{-1}$ is only $\sim 0.4 \%$.
\end{abstract}

Subject headings: ISM: structure - supernova remnants - infrared: ISM

\section{Introduction}

The composition and structure of the interstellar medium (ISM) are critical to our understanding of the evolution of stars and galaxies. The gas is shaped by turbulence and magnetic fields and is highly structured (Verschuur 1995). Instabilities cause the gas to collect into compact filaments and sheets that collapse into stars (e.g., Vázquez-Semadeni et al. 2007 and references therein; Boss 2005). The interstellar grains account for only $1 \%$ of the total mass of the ISM, but their interaction with stellar photons is the dominant source of opacity and hence strongly influences star formation and evolution. There have been several observations that give us some clues to the nature of the dust in the ISM: for example, the behavior of Polycyclic Aromatic Hydrocarbons (PAHs) in nearby galaxies (Smith et al.

\footnotetext{
${ }^{1}$ Steward Observatory, University of Arizona, 933 North Cherry Avenue, Tucson, Arizona 85721

${ }^{2}$ currently at the Department of Astronomy and Astrophysics, The University of Chicago, 5640 S. Ellis Ave., Chicago, Illinois 60637

${ }^{3}$ Max-Planck-Institut für Astronomie, Königstuhl 17, 69117 Heidelberg

${ }^{4}$ Astronomy Department, University of Virginia, P. O. Box 3818, Charlottesville, VA 22903-0818
} 
2007), and the mass or size distribution of dust particles in the ISM (Kim \& Martin 1996; Mathis et al. 1977), to just name a few (see review by Draine 2003). However, as yet, no fully developed model has been developed for the detailed morphology of the ISM.

An understanding of the ISM structure is necessary to understand the forces and mechanisms that shape it and set the preconditions for star formation and other widely studied processes (e.g., Carlqvist \& Gahm 1992; Verschuur 1995; Fiege \& Pudritz 2000). The ISM structure is also a key input to studies of radiative transfer, and therefore is critical to understanding the properties of dust-embedded sources (e.g., Bruzual et al. 1988; Witt \& Gordon 1996; Wolf et al. 1999; Hegmann \& Kegel 2003). Specific examples include young stellar object clumpy circumstellar media (e.g., Indebetouw et al. 2006), HII regions (e.g., Wood et al. 2005), and galaxy disks (e.g., Inoue 2005) and references in these works. It has been shown that the assumed structure can affect the wavelength dependence of the attenuation (e.g., Fischera \& Dopita 2005) and can significantly modify the observed spectral energy distribution of a source (Indebetouw et al. 2006). Clumping can also allow self-shielding of the ISM gas and therefore can alter observed line strengths (e.g. Burton et al. 1990).

The structure of the ISM is conventionally measured using HI observations. Verschuur (1995) reviews these results, emphasizing the prevalence of filamentary structures and the possible causes for them. HI data can identify such features through coherent velocity structures, but it is difficult to relate them accurately to one another along the line of sight. Braun \& Kanekar (2005) have recently used HI measurements to discover tiny interstellar clouds in the local ISM, with dimensions of $0.1 \mathrm{pc}$ or even less. There have been several efforts to study the fractal structures of the ISM (Elmegreen \& Falgarone 1996) on subparsec scales (Ingalls et al. 2004; Falgarone et al. 2004, and references therein). Small scale structures have also been probed by observing the scintillation of signals from compact radio sources, indicating that the density fluctuation spectrum is consistent with Kolmorgov turbulence (e.g., Stinebring et al. 2000). More closely related to our study are three-dimensional maps of the dust structures in the foreground of supernova (SN) 1987A (Xu et al. 1995) and of type Ia SN in general (Patat 2005). These studies are based on the highly complex physical characteristics of interstellar dust particles, such as three-dimensional multiple scattering or polarization, with dependencies on both the dust parameters and the geometry. Thus, they must introduce additional parameters that are difficult to constrain and can increase the uncertainties in the results.

Here we present a simple and straightforward method to study the structure of the ISM surrounding the supernova remnant (SNR) Cassiopeia A (Cas A) at a resolution of 0.1pc. Cas A exploded approximately 10,000 years ago, and the light coming directly from the explosion reached Earth about 325 years ago (Thorstensen et al. 2001). In previous 
work (Krause et al. 2005), we discovered infrared (IR) features at $24 \mu \mathrm{m}$ up to about 25 arcmin from the center of the SNR. These features were apparently moving at superluminal velocities, much faster than the velocity of fast-moving knots around the SNR. Although initially some features appeared to be associated with a more recent event in the core of the SNR, further tracking of their motions now makes this explanation appear unlikely. We

believe that all the observed IR features are the echoed reradiation of thermal energy as the light from the Cas A supernova passes through its surrounding ISM and heats the dust. Because of the indirect path to reach us and the resulting increased propagation time, we see these features now. These IR-radiating features, called IR echoes, are a novel approach to explore the distribution and the morphological structure of the ISM in three dimensions, and thus they solve the limitations of the previous integrated measures of the ISM structure in two dimensional, projected images. In this paper, we describe the geometry of the echoes in $\S 2$, present observations of them over nearly a three year interval in $\S 3$, develop and apply the tools to analyze the data and construct images of the ISM in $\S 4$, discuss the results in $\S 5$, and summarize our conclusions in $\S 6$.

\section{Background}

IR echoes arise when the spherical pulse of electromagnetic radiation from the SN explosion encounters and heats interstellar dust. The resulting IR radiation traces the ISM along the radiation pulse. The IR echoes we observe at a given time travel over the same total distance from Cas A to dust grains in the ISM and then to the Earth, which requires that the echoes lie on an ellipsoid whose foci are Cas A and the Earth. Thus in a sequence of observations, the IR echoes must move successively outward to more distant ellipsoidal regions of the ISM. We then view continuously changing circular cross-sections of the ellipsoids as the light passes through interstellar space.

By assuming that the distance to the SNR from the Earth is much greater than the distance between the observed echo and the source, the ellipsoidal structure can be simplified to a paraboloid (see Fig. 1.). The paraboloid that forms the locus for the echoes at any time is defined by the set of points such that the light travel distance from the supernove to the vertex and then back to the observer, $\mathrm{D}+\mathrm{ct}$, equals the light travel distance to any point on the paraboloid (light travel distance $r$ ), and then to the observer (light travel distance $\mathrm{D}-\mathrm{z})$.

$$
D+c t=(D-z)+r,
$$




$$
\begin{gathered}
h^{2}+z^{2}=r^{2}, \\
z=\frac{1}{2}\left(\frac{h^{2}}{c t}-c t\right),
\end{gathered}
$$

where $D$ is the distance between the SNR and the Earth, $z$ is the distance of the observed IR echo from the SNR with the positive direction toward the observer, $r$ is the distance from the SNR to the heated ISM dust (Fig. 1 demonstrates that the echoes seen at any one time have a range of distances from the SNR), $h$ is the perpendicular distance of the echo to the line of sight between Cas A and the Earth, and $t$ represents the time difference between the observed outburst of Cas A and the echo observation date.

\section{Observations}

Between 2003 November 30 and 2006 October 2, we used the Multiband Imaging Photometer for Spitzer (MIPS; Rieke et al. 2004) to obtain 11 images at $24 \mu \mathrm{m}$ of Cas A in six epochs with an interval of about 6 months between epochs. Figure 2 shows one such image. The first 5 images (IOC Campaign W, PID 231, PID 233, PID 718, PID 20381) were centered on Cas A and observed in scanning mode, while the other 6, all at a single epoch, were taken in photometry mode (PID 30571), concentrating on a number of selected regions. The first two images (epochs 1 and 2, 2003 November 30 and 2004 December 2) were taken within a region about 52 arc min long by 12 arc min wide, the third image (epoch 3, 2005 February 3) about 120 arc min long by 120 arc min wide, the next two images (epochs 4 and 5, 2005 September 1 and 2006 February 14) about 200 arc min long by 200 arc min wide; the last six images (epoch 6, 2006 October 2) are each about 20 arc min long and 20 arc min wide.

\section{Data Processing and Analysis}

Initial reductions of these data used standard procedures centered around the MIPS Instrument team Data Analysis Tool (DAT) (Gordon et al. 2005). Fine-scale moving features in the reduced images behave as predicted for IR light echoes in $\S 2$. For example, the most distant such features are at a projected distance of about $100 \mathrm{pc}$ from the site of the SN, and many of them have arc-like morphologies pointing back toward the SNR. 
The reconstruction of the three-dimensional structure of the ISM from the IR echoes requires a number of additional data analysis steps to isolate the IR echoes from other features. They were performed in the following order: fast Fourier transform (FFT) filtering $(\S 4.1)$, star cleaning $(\S 4.2)$, subtraction of images $(\S 4.3)$, and finally determination of the three-dimensional structure of the ISM $(\S 4.4)$.

\section{1. $\quad$ Fast Fourier Transform (FFT) Filtering}

In addition to the fine filamentary structures, the IR images include strong extended emission. The extended structures resemble the diffuse galactic cirrus emission and appear to be more than 40 arcmin in scale. In comparison, the fine structures due to infrared echoes stand out distinctly against the diffuse sky background but are fainter than many stars and nebular components. Due to the characteristic geometry (see Fig. 1), the motions of the IR echoes projected close to the position of Cas A are fore-shortened and hence those echoes are generally observed to move more slowly than the echoes far out near the edges of our images. The typical estimated apparent motion near Cas A is about 2 arc sec within a year compared with about 18 arc sec at a position perpendicular to the center of Cas A relative to the line of sight. The bottom four panels in Fig. 2 show the changes in morphology of some of the IR echoes for about one and a half years of observations from epoch 3 to epoch 6.

The echoes can be isolated by taking differences of images at different epochs. Because of the limited time interval covered by our observations, such images can reveal stucture



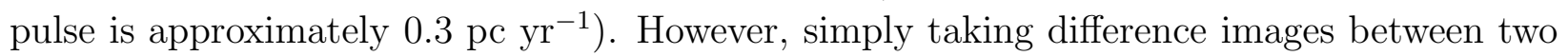
consecutive epochs is not the optimum way to eliminate the sky background underneath or in front of the IR echoes. As the Spitzer telescope moves in space, it looks through various zodiacal cloud columns depending on the observing date, changing the level of zodiacal foreground emission. In addition, the echo features do not necessarily move far enough over our measurement period for us to measure the structure of the fixed background emission behind them. Therefore, low frequency background structure can distort our measurements of the echoes.

To reduce these two effects, we use high pass filtering to suppress the spatial frequencies where the information on the ISM is compromised by our limited time range of observation anyway. To set the filter parameters, we took a difference image of subregions of epoch 3 and epoch 5. We selected these epochs to have large areal coverage (20 arcmin square) over a reasonably long time baseline (1 year). We computed the radial power spectrum for the 
difference image by azimuthally averaging in equally spaced wavenumber bins. Fig. 3 shows a shaded surface representation of the logarithm of the power spectrum of the difference image on the left and the averaged power spectrum on the right. As expected, the power spectrum rolls over below 1.5 cycles $\mathrm{pc}^{-1}$, which corresponds to the lowest frequency at which echoes observed over a 1 year period should produce signals in the difference image. Since our total observation set covers three years, we set the filtering to $\sim 0.5$ cycles pc $^{-1}$, so we can insure almost 100\% transmission of the echo information (See Fig. 3).

For computational convenience, each epoch image was disaggregated to form small square subregions of 1001 x 1001 pixels, avoiding the bright emission within about 8 arcmin of Cas A. To eliminate the diffuse sky background emission, the high-pass filter was then applied to each small region with a cutoff frequency of 0.01 cycles per pixel, or 10

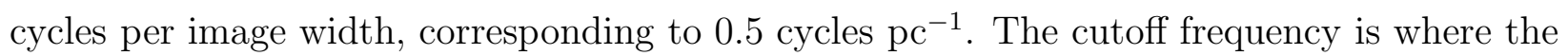
power transmitted by the high pass filter reaches its half maximum (see Fig. 3). The filter was implemented using the FFT routine in Interactive Data Language (IDL) and applying a Butterworth high pass filter. The equation for this filter is

$$
\text { high_pass }=1.0 /\left(1.0+\left((\text { cutof } f / \text { distfun })^{(2 * o r d e r ~}\right),\right. \text {, }
$$

where cutoff is the predetermined value meant to represent the threshold frequency, 0.01 pixel $^{-1}$, above which the filter passes information, the order parameter determines the steepness of the filter; the higher the order, the steeper the band, and distfun is an array of the high pass filter image before any adjustment is made. The value of order was set to 1 to avoid possible artifacts due to the effect of the high filter order on the frequency response. The cutoff frequency was kept consistent in all images to retain properly scaled images for subtraction later $(\S 4.3)$.

With the application of this filter, almost all of the diffuse background emission was satisfactorily removed, only leaving spatially varying sources such as IR echoes, plus stars and bright nebulosity.

\subsection{Star Cleaning}

Depending on when the observations were scheduled, the position angle (PA) of the Spitzer telescope may vary. At the Ecliptic latitude of $\sim 54^{\circ}$ for Cas A, there is a change in PA by between $0.5^{\circ}$ and $1^{\circ}$ per day. As a result, the relation of a star to its latent image and other artifacts changes. This effect requires that we remove point sources before subtraction of the images, thus minimizing any possible star residuals. 
Stars were located by a set of IDL DAOPHOT procedures. The first procedure of the set, find, detected stars that passed the user-given criteria of $\mathrm{FWHM}=4.0$ and threshold intensity $=1.0$ in pixel values. We ran the find routine with different trials of FWHM ranging from 3.0 to 6.0 until we settled on 4.0 as the typical star FWHM that detected most of the stars in our images. The threshold intensity was set to 1.0; the stars whose central intensity was less than 1.0 were suppressed close to zero in difference images (§4.3). After the stars had been located, aperture photometry was used to measure the magnitude of each star and the background. Several reference stars in each image were manually selected to define a point-spread function (PSF) and then the best estimated PSF was subtracted from every detected stellar image.

Although most of the stars were eliminated by this method, some star residuals were still present, especially for the extremely bright stars whose visible images extend to more than 10 pixels. These bright stars were interactively suppressed to a low pixel value $=0.1$ by subtracting the PSF, and later they were removed almost completely by image subtraction (§4.3). The scanning process with MIPS produced a series of quasi-star small dots from latent images of bright stars along the scanning direction. In each observing period, scanning was performed in a different direction, so using the same star position for all the different epochs did not remove the latent images. Thus, we created separate star position lists and applied them for those images that had the problem. Another artifact due to the bright point sources was vertical pinstripings, also known as jailbars, as a result of pixel saturation. Faint jailbars were cleared up by subtraction ( $\$ 4.3)$, but interactive cleaning was performed for strong jailbars, based on local averages of the repetitive pattern.

\subsection{Subtraction}

Once a light pulse is absorbed by dust grains, the IR light echoes are re-emitted quickly into successive paraboloids and can be identified via a change in either the apparent motion or pixel values. Especially for those whose changes in apparent position are too small to be seen by eye - for example the echoes coming from the same radial direction at different times - the pixel intensity change can be the better way to probe them.

To implement this approach, we performed the following steps. First, the below-zero pixels in star-cleaned images were scaled to zero; we are only interested in the positive signals, and removing any negative pixel points will prevent any misinterpretation of the results after subtracting images. Then, two images that covered the same region but were taken on different dates were subtracted from each other. Echoes in the first image will appear as positive pixel values, while those in the subtracted image will yield negative pixel 
values (see Fig. 4). However, there may also be residuals due to the fluctuation of intensity in the background level itself due to the varying position of Spitzer in its orbit. To deal with this possibility, we took a difference image of a region far away from any apparent echo structures in the two epochs. Several regions were tested, and the maximum difference-pixel residuals for each trial region were measured and found on average to be approximately within \pm 0.7 in units of pixel values. Therefore, any points whose pixel values in the difference image are between \pm 0.7 were considered as possibly coming from the diffuse background and rejected. Most of the low-pixel-valued echoes were then recovered; this approach enabled us to also trace echoes lying above/below the nebulosity.

\subsection{Three-dimensional Structure of the ISM}

To produce three-dimensional maps of the ISM, we first set the center of Cas A to the reference point $(0,0,0)$ in a three dimensional space with positive $x$ toward the west of Cas A, positive $y$ toward the north, and positive $z$ toward the observer. We computed the two dimensional projected coordinates, $x$ and $y$, of the echoes that pass the threshold, \pm 0.7 (§4.3). With the resulting $x$ and $y$ values, the projected distance from Cas A to the echoes in the plane of the sky, $h$ in Eq. (2), was calculated using the Pythagorean theorem for every point.

We adopted the explosion date of Cas A to be A.D. $1671.3 \pm 0.9$ (Thorstensen et al. 2001). Solving for the spatial distance, $r$ in Eq. (11) and substituting it into Eq. (2) gives the line-of-sight depth, $z$ in Eq. (3). We recorded the echoes using the 3-D plot procedure, XPLOT3D, in IDL. This procedure takes three vectors in $x, y$, and $z$ coordinates and plots them as scattered data points in three dimensions. Fig. 5 illustrates this process for images from the first two epochs of our observational series. Fig. 6 shows the final three-dimensional image of echoes from all six epochs. The images are constructed in pointillist style, consisting of dots representing each position where an echo was detected. No attempt is made to encode the intensity of the echoes. To distinguish data points taken on different observing dates, different symbol colors are used.

\section{Discussion}

In this section, we first show that the energy pulse from the SN explosion has overtaken residuals from the progenitor massive star and its episodes of mass loss. As a result, the IR echoes reveal the structure of undisturbed ISM, which we then discuss. 


\subsection{Three Possible Sources of IR-Emitting Dust}

We consider three possible origins for the dust probed by the IR light echoes: newly condensed SN ejecta, circumstellar material (CSM) produced by stellar winds as the progenitor star of Cas A evolved until its death by its supernova explosion, and the pre-existent ISM dust.

High-velocity SN ejecta from Cas A have previously been identified with expansion speeds up to nearly $12,000 \mathrm{~km} \mathrm{~s}^{-1}$ (Fesen 2001). Since the IR echoes lie well outside even the most rapidly moving ejecta, at positions consistent with their moving outward at the speed of light, we can exclude that they are associated with such ejecta.

We have also considered the possibility that the echoes could arise from the circumstellar dust released during stellar evolutionary phases. García-Segura et al. (1996) showed that the progenitor star of Cas A underwent the evolutionary sequence O-star, Red-Supergiant (RSG) star, and Wolf-Rayet (WR) star roughly $10^{4}$ yr before its supernova explosion; they constrained its initial mass to be between $25 M_{\odot}$ and $35 M_{\odot}$.

In a $35 M_{\odot}$ star model, the wind velocity is estimated at a few $1000 \mathrm{~km} \mathrm{~s}^{-1}$ both in the main sequence (MS) and WR stages, and falls to $<100 \mathrm{~km} \mathrm{~s}^{-1}$ in the RSG stage (GarcíaSegura et al. 1996; Dwarkadas 2004). About 70\% of the total material loss in the wind from MS to WR stages is lost in the RSG stage. With a small wind velocity, a high wind density, and a low temperature, molecule and dust formation is likely in the RSG stage, but the RSG thin shell expands only up to $<10 \mathrm{pc}$, due to its relatively slow wind velocity $\left(\sim 75 \mathrm{~km} \mathrm{~s}^{-1}\right)$. Thus, the main contributor to pushing the high density RSG materials outwards from the center of the star is the strong WR wind with its wind velocity $\sim 1000 \mathrm{~km} \mathrm{~s}^{-1}$ lasting $10^{4}$ yrs. The study of pulsations in RSG with high L/M ratio by Heger et al. (1997) also concluded pre-supernova stars go through a phase of violent pulsational instability several $10^{4} \mathrm{yr}$ before the supernova explosion, which might add to the WR wind.

The upper limit for a mass loss shell boundary radius has been estimated at $\sim 38 \mathrm{pc}$ for an ambient ISM density of $20 \mathrm{~cm}^{-3}$ (García-Segura et al. 1996) or at $\sim 74$ pc for an ISM density of $1 \mathrm{~cm}^{-3}$ (Dwarkadas 2004). To understand whether this boundary lies inside or outside of the IR echoes requires that we estimate the ISM density around Cas A. Krause et al. (2004) find a typical column density in gas of $\sim 40 \mathrm{mg} \mathrm{cm}^{-2}$ in front of the SNR. Assuming this gas is distributed uniformly between us and the SNR would result in an

estimate of $\sim 2 \mathrm{~cm}^{-3}$ for the average ISM density around Cas A. However, the line of sight to the SNR intersects the Perseus Arm, the outermost main spiral arm of the Milky Way, and most of the material should also lie in this arm rather than in the space between it and the sun (Wilson et al. 1993). A typical width of a Milky Way arm is $1.32 \mathrm{kpc}$ (Russeil 2003). 
We can therefore assume that most of the interstellar material lies within $1 \mathrm{kpc}$ of the SNR, compared with its distance of $3.4 \mathrm{kpc}$. Thus, a plausible estimate for the ISM density in

the space around Cas $\mathrm{A}$ is $\sim 6 \mathrm{~cm}^{-3}$. Consequently, for average conditions, the boundary of the shell should lie at or within the $50 \mathrm{pc}$ radius where the vertex of the paraboloid corresponding to epoch 1 is located and the IR echoes are observed at the shortest distance since the explosion of Cas A. In most locations, this argument indicates that the echoes should probe undisturbed ISM.

To expand the argument, the ISM density varies substantially in different directions from Cas A (as we show below), so we would expect the wind to have penetrated to varying depths around $50 \mathrm{pc}$. Yet there is no change in the character of the IR echoes. Therefore, the stellar wind is unlikely to have penetrated to the ISM and modified it where the IR echoes are produced.

\subsection{The Three-dimensional properties of the ambient ISM}

\subsubsection{Morphology}

Fig. 6 displays a three-dimensional picture of the distribution of dust surrounding Cas A. The light echoes display the structure of the ISM dust probed in the form of multiple sheet-like surfaces. Due to the interval of six months between successive epochs (except epoch 1 and epoch 2 with about one year time difference and epoch 2 and epoch 3, separated by about two months), the sheets are separated by about $0.1 \mathrm{pc}$.

Although the echoes are distributed all around the SNR, they are also highly structured. We made a two-dimensional plot of the echoes, or dust particles, projected onto the plane of sky to demonstrate the asymmetrical density distribution of dust (see Fig. 7). We see a higher density of dust on the west and northwest sides of Cas A than elsewhere.

To examine the morphology of the ISM more closely, the same small region in the images at the bottom of Fig. 2 is plotted in three dimensions in Fig. 8. This figure demonstrates that the ISM is distributed in very well-defined filaments and sheets with typical dimensions larger than our resolution limit of $\sim 0.1 \mathrm{pc}$, but only slightly so in the narrowest dimensions.

The three-dimensional structure around Cas A revealed by the IR echoes shows the position, density, and shape of dust clouds on a scale of the observational limit of $0.1 \mathrm{pc}$ over a region with projected radius of $\sim 50 \mathrm{pc}$ (see Fig. 6). We now use this information to determine some general properties of the ISM surrounding Cas A. 


\subsubsection{Filling Factor}

In Fig. 9, we plot the filling factor of the small-scale ISM clumps in epoch 4 as a function of the projected distance, $h$, within a range of 9 to 109 parsecs and with a bin size of 10 pc, perpendicular to the line of sight. The SN light pulse slices the ISM in thin sheets, so the filling factor is readily computed as the fraction of each sheet containing echoes. The two-dimensional coordinate system was divided into four quadrants, which thus yields four equal areas of the echo-generating paraboloid. In each quadrant, the percent ratio of the areas of echoes and of the portion of the paraboloid was computed within the above distance range (see Fig. 9). The filling factors are generally higher on the west side of Cas A, as can also be easily seen from Fig. 7. The filling factors tend to fall with increasing distance from the SNR. This overall behavior suggests that most of the dust lies behind and relatively near to Cas A. It is also possible that the attenuation of the intensity of the outgoing pulse of electromagnetic radiation, due to extinction as it propagates through the ISM, gives rise to the fainter IR light echoes at greater distances.

The average filling factor of $\sim 0.4 \%$ is overplotted as a dashed line. This value is lower than but consistent with the filling factor, $\sim 1 \%$, determined by Krause et al. (2005) from the comparison of the net luminosity observed with predictions for a flare of isotropic energy at the northern lobe of Cas A. Their estimation covered a length span of 6 arcsec, while ours covers about 40,000 square arcmin, so ours provides a more representative value. This result emphasizes the extreme clumpiness of the ISM at scales above the wavenumber $k \sim$ 0.5 cycles $\mathrm{pc}^{-1}$ with substantial portions concentrated into a very small volume.

\subsubsection{Mass Estimation for the Warm Dust in small ISM Features}

The average spectral energy distribution (SED) of 59 selected infrared features in the northern lobe region of Cas A shows an increasing surface brightness with wavelength from 2.2 to $24 \mu \mathrm{m}$ (Krause et al. 2005). Images taken at 70 and $160 \mu \mathrm{m}$ exhibit no distinct echo features but display overall bright cirrus background emission. It is possible that the farinfrared (FIR) images may have cool echo features, but they are not resolvable due to the dominant cool diffuse background clouds. Thus, we assume that the IR light echoes observed at $24 \mu \mathrm{m}$ are mostly coming from warm dust grains. Because the SED is available over a small wavelength range, we cannot determine the exact blackbody energy distribution of the dust around Cas A, but we can assign an upper limit of 100 K to the temperature of the dust by taking $24 \mu \mathrm{m}$ as the peak wavelength.

The total flux of the IR light echoes was computed and used to deduce the mass of the 
heated dust within successive parabolic volumes defined by epochs 4 and 5 . The dust mass is determined from the $24 \mu \mathrm{m}$ emission by (Marleau et al. 2006):

$$
M_{d}=\frac{S_{\nu} D^{2}}{\kappa_{d} B_{(\nu, T)}},
$$

where $S_{\nu}$ is the total flux of echoes from epoch 4 and epoch $5, D$ is the distance to the dust, $\kappa_{d}$ is the mass absorption opacity of the dust, for which a value of $492 \mathrm{~cm}^{2} \mathrm{~g}^{-1}$ at $24 \mu \mathrm{m}$ is assumed ( $\mathrm{Li} \&$ Draine 2001), and $B_{(\nu, T)}$ is the Planck function. The total mass estimate for dust within the volume of the small echo features is $\sim 0.014 \mathrm{M}_{\odot}$, yielding a gas mass of $\sim$ $1.4 \mathrm{M}_{\odot}$ for a gas-to-dust mass ratio of 100 . Since this estimate is based on an upper limit for the dust temperature, the mass is a lower limit. The full volume occupied by the echoes and surrounding space for this determination is $3 \times 10^{5} p c^{3}$, and the average (lower limit) ISM density represented by the echoes is therefore only $3 \times 10^{-25} \mathrm{~kg} \mathrm{~m}^{-3}$.

We have tested whether the estimated mass is affected by stochastic heating. We ran heating simulations for the SN echo using silicate grains. For these runs, we assumed that the echo was the result of the SN explosion, that the SN had a bolometric luminosity of $5.0 \times 10^{9} \mathrm{~L}_{\odot}$ (Young et al. 2006), and that the echoes are at a distance of $67 \mathrm{pc}$ from the SNR. We adopted the SED of SN 1987A at day 100 (Arnett et al. 1989). The grain sizes and their heating and cooling times are listed in Table 1 . The heating time is the average interval between photon absorptions.

As can be seen in Table 1 , even at $20 \AA$ the grain will usually be hit by a photon with substantial energy well before it can cool significantly. The only grains that will show some stochastic heating will be $\leq 15 \AA$ in size. Other grain types (e.g., graphite) do not differ in heat capacity and radiative properties sufficiently to modify the size range substantially (e.g., Dwek 1986). Such small grains will not emit efficiently at $24 \mu \mathrm{m}$. Thus, it is very unlikely for the grains around Cas A to be heated stochastically to a sufficient extent to undermine our mass estimate.

\subsubsection{The Fractal Nature of the ISM and the Total Mass Estimation}

There is a growing consensus that turbulence plays a major role in the dynamics of molecular clouds and results in fractal structures (Falgarone \& Phillips 1990; Elmegreen \& Falgarone 1996). Fractals are fragmented geometric shapes that show self-similarity at all levels of magnification (Mandelbrot 1983).

To test the fractal nature of the structures, we selected two echo-dominant regions 
observed with a time interval of one year. We computed the two-dimensional power spectrum of the difference image (Fig. 3). Since it is roughly symmetric around the central peak, we converted it to a one-dimensional power spectrum by azimuthal averaging. We used several subregions of the Spitzer mosaics with no detectable emission as noise images, and analyzed them in the same way. The individual power spectra from the noise images were summed and averaged, and the averaged noise power spectrum was subtracted from the power spectrum of the original image. Since the difference image of adjacent epochs isolates a thin layer of the ISM from the full depth of the ISM surrounding the SNR, the resulting power spectrum minimizes effects due to the superposition of regions along the line of sight.

The power spectra (original, noise, and noise-subtracted) of two sample regions are compared in Fig. 10. The power spectrum from the first region displays a slight transition of the slope at the frequency of $\sim 2.0$ cycles $\mathrm{pc}^{-1}$, most likely due to the decrease in intensity of the components of the features that are almost invariant over the time difference of one year. That assumption becomes more certain because the power spectrum from the second region shows less of a distinct transition of the slope. The second region is located further from the line of sight to the SNR than the first; thus, the spatial change in the time difference in the second region results in a greater apparent motion in two dimensional images. For both regions, the behavior of the noise-subtracted data is reasonably consistent with a power law, $\mathrm{k}^{-\beta}$ with an index of $\beta=3.5$. This slope was found by Ingalls et al. (2004) for a $24 \mu \mathrm{m}$ image of the general ISM. This behavior is as expected for fractal structure. That is, our three-dimensional images confirm the fractal structure of the ISM previously deduced from studies in only two dimensions.

Assuming that the power spectrum of the two regions is described by the power-law behavior with the spectral index 3.5 over the low frequency range from $\sim 10^{-3}$ to 1.0 cycles $\mathrm{pc}^{-1}$ (as indicated by Ingalls et al. (2004) in the wavenumber range from $\sim 10^{-3}$ to $10^{-1}$ ),


result is $\sim 6.2 \times 10^{-23} \mathrm{~kg} \mathrm{~m}^{-3}$, about 200 times more than in the small $\left(\mathrm{k}>0.5\right.$ cycles pc $\left.^{-1}\right)$ features alone. The derived total density is slightly higher than the average mass density of dust in the diffuse ISM of $\sim 1.8 \times 10^{-23} \mathrm{~kg} \mathrm{~m}^{-3}$ (Whittet 2003). Given the uncertainties, particularly in the dust temperature, this value agrees well with expectations (e.g., §5.1). A corollary of the modest density associated with the echoes is that they are optically thin to the supernova radiation and that we are measuring volume emission, not just that from a surface layer. 


\subsubsection{Comparison with Previous Work}

Complexes of filamentary and wavy knots have been found in many observations of the small-scale ISM environment. Examples include the Chamaeleon III region (Gahm et al. 2002), elephant trunk structures (Carlqvist et al. 1998), the Lagoon nebula (Caulet 1997), diffuse molecular clouds (Falgarone et al. 2004), and a nearby cold interstellar cloud (McClure-Griffiths et al. 2006). Typically, ISM filaments are distributed in long, finger-like patterns and are observed overlapping and crossing each other due to projection effects. These overlapping filaments can be identified by differences in radial velocity and in color intensity at the observed wavelength, e.g., the contrast between dark and bright filaments.

The individual elongated filamental features in Fig. 7 are reminiscent of those studied in two dark clouds, the Lynds 204 complex and the Sandqvist 187-188 complex, by Carlqvist and Gahm (1992). They suggested that the Bennett pinch, resulting from a cylindrical plasma carrying an axially directed current, could be the physical mechanism for formation of the wavy filaments. In this process, the plasma undergoes a pinch compression by the current simultaneously with the generation of a toroidal magnetic field. Verschuur (1995) has reviewed structures observed with high resolution HI data. He also suggests the pinching action of electric currents as contributing to the highly filamentary structures of the ISM. Detailed numerical models invoking such processes have been described by Fiege and Pudritz (2000).

It is not clear whether the filaments are stable or unstable. The generalized Bennett relationship for the equilibrium of a filament consists of two inward pinching forces, due to the current and to gravity, and three outward forces due to gas pressure, to the axial magnetic field, and to rotational energy around the filament axes (Verschuur 1995). There is a possibility that the pinching action undergoes various kinds of instabilities and develops dynamically. Carlqvist and Gahm (1992) discuss two such examples: 1.) kink instability, when motions in a conduction fluid body change the initial magnetic field in a way that it becomes unstable and produces a kink (Alfvén and Fälthammar 1963); and 2.) sausage instability, which originates from a small radial disturbance of a pinched filament and may lead to disruption of the filament. Comparisons of the wavy shapes of the filaments in our data with the predictions of such models can be used to test theoretical hypotheses for filament formation and maintenance.

Molecular clouds have also been described as fractal and highly dynamical (Falgarone et al. 2004). Fractal structures are characterized by a power law power spectrum of the image $\left(\propto \mathrm{k}^{-\beta}\right)$ and a completely random distribution of the image phases (Stutzki et al. 1998). Several studies have obtained power spectra of the ISM that show the characteristic fractal self-similar structure, with the spectral indices $\beta \sim 2.6$ to 2.8 (Green 1993) or $\beta \sim 2.6$ to 
3.5 (Ingalls et al. 2004). Stutzki et al. (1998) introduced the 2-dimensional $\Delta$ - variance method, which has been used to find $\beta \sim 2.5$ (Bensch et al. 2001; Falgarone et al. 2004, and references therein). We show that the power spectrum found by Ingalls et al. (2004) with $\beta=3.5$ is consistent with the three dimensional ISM structure, and we can place a physical scale on the structures where we have fitted it.

\section{Conclusion}

IR light echo observations at $24 \mu \mathrm{m}$ have allowed the exposition of the three-dimensional physical structure of the ISM around the SNR, Cas A. From high pass filtering to subtraction, we have shown the detailed analysis required to distinguish as many IR light echoes as possible from any diffuse background and other fixed sources, such as stars and bright nebular emission.

From our results, we draw the following main conclusions:

- The detailed behavior of the IR light echoes confirms that they are re-radiation of the absorbed outward-moving SN light pulse by the ISM surrounding Cas A.

- The three-dimensional display of the echoes shows different sizes of clumps connected with filaments that trace sheet-like patterns.

- The surface filling factor of the heated dust grains is about $0.4 \%$, for fine structures (spatial frequencies $\geq 0.5$ cycles $\mathrm{pc}^{-1}$ ).

- The structures have a power-law power spectrum $\mathrm{k}^{-\beta}$ with index $\beta \sim 3.5$, consistent with fractal structure.


we have studied is $\sim 3 \times 10^{-25} \mathrm{~kg} \mathrm{~m}^{-3}$. If we integrate the power spectrum down to $\sim 0.01$ cycles $\mathrm{pc}^{-1}$, we estimate that the ISM surrounding Cas A is $\sim 6 \times 10^{-23} \mathrm{~kg} \mathrm{~m}^{-3}$, more dense than the general diffuse ISM by a factor of a few.

This work is based on observations made with the Spitzer Space Telescope, which is operated by the Jet Propulsion Laboratory, California Institute of Technology under contract

with NASA. Support for this work was provided by NASA through JPL/Caltech contracts 1276396 and 1255094 to the University of Arizona. 


\section{REFERENCES}

Alfvén \& Fälthammar, C.-G. 1963, Cosmical Electrodynamics (2nd ed.; Oxford: Carendon)

Arnett, W. D., Bahcall, J. N., Kirshner, R. P., \& Woosley, S. E. 1989, ARA\&A, 27, 629

Bensch, F., Stutzki, J., \& Ossenkopf, V. 2001, A\&A, 366, 636

Boss, A. P. 2005, ApJ, 622, 393

Braun, R., \& Kanekar, 2005, A\&A, 436L, 53

Bruzual, A. G., Magris, C. G., \& Calvert, N. 1988, ApJ, 333, 673

Burton, M. G., Hollenbach, D. J., \& Tielens, A. G. M. 1990, ApJ, 365, 620

Carlqvist, P., \& Gahm, G. F. 1992, IEEE Trans. Plasma Sci. 20, 867

Carlqvist, P., Kristen, H., \& Gahm, G. F. 1998, A\&A, 332, L5

Caulet A. 1997, ST-ECF Newslett., 24, 12

Draine, B. T. 2003, ARAA, 41, 241

Dwarkadas, V. 2004, in Cambridge contemporary astrophysics ser., Cosmic explosions in three dimensions : asymmetries in supernovae and gamma-ray bursts, ed. P. Hoflich, P. Kumar and J. C. Wheeler, (Cambridge, UK: Cambridge University Press), 74

Dwek, E. 1986, ApJ, 302, 363

Elmegreen, B. G., \& Falgarone, E. 1996, ApJ, 471, 816

Falgarone, E. \& Phillips, T. G. 1990, ApJ, 359, 344

Falgarone, E., Hily-Blant, P., \& Levrier, F. 2004, Ap\&SS, 292, 89

Fesen, R. A. 2001, ApJ, 133, 161

Fiege, J. D., \& Pudritz, R. E. 2000, MNRAS, 311, 85

Fischera, J. \& Dopita, M. 2005, ApJ, 619, 340

Gahm, G. F., Lehtine, K., Carlqvist, P., Harju, J., Juvela, M, \& Mattila, K. 2002, A\&A, 389,577

García-Segura, G., Langer, N., \& Mac Low, M.-M. 1996, A\&A, 316, 133 
Gordon, K. D., et al. 2005, PASP, 117, 503

Green, D.A. 1993, MNRAS, 262, 327

Heger, A., Jeannin, L., Langer, N., \& Baraffe, I. 1997, A\&A, 327, 224

Hegmann, M. \& Kegel, W. H. 2003, MNRAS, 342, 453

Indebetouw, R., Whitney, B. A., Johnson, K. E., \& Wood, K. 2006, ApJ, 636, 362

Ingalls, J. G., et al. 2004, ApJSS, 154, 281

Inoue, A. K. 2005, MNRAS, 359, 171

Kim, S.-H., \& Martin, P. G. 1996, ApJ, 462, 296

Krause, O., Birkmann, S. M., Rieke, G. H., Lemke, D., Klaas, U., Hines, D. C., \& Gordon, K. D. 2004, Nature, 432, 596

Krause, O., et al. 2005, Science, 308, 1604

Li, A., \& Draine, B. T. 2001, ApJ, 554, 778

Mandelbrot, B. B., 1983, The Fractal Geometry of Nature, Freeman, San Francisco.

Marleau, F. R., et al. 2006, ApJ, 646, 929

McClure-Griffiths, N. M., Dickey, J. M., Gaensler, B. M., Green, A. J., \& Haverkorn, M. 2006, ApJ, 652, 1339

Patat, F. 2005, MNRAS, 357, 1161

Rieke, G. H., et al. 2004, ApJS, 154, 25

Russeil, D. 2003, A\&A, 397, 133

Smith, J. D. T., et al. 2007, ApJ, 656, 770

Stinebring, D. R., Smirnova, T. V., Hankins, T. H., Hovis, J. S., Kaspi, V. M., Kempner, J. C., Myers, E., \& Nice, D. J. 2000, ApJ, 539, 300

Stutzki, J., Bensch, F., Heithausen, A., Ossenkopf, V., Zielinsky, M., 1998, A\&A, 336, 697

Thorstensen, J. R., Fesen, R. A., \& van den Bergh, S. 2001, ApJ, 122, 297 
Vázquez-Semadeni, E., Gómez, G.C., Jappsen, A.-K., Ballesteros-Paredes, J., González, R.F., Klessen, R.S., 2007, ApJ, 657, 870

Verschuur, G. 1995, Ap\&SS. 227, 187

Whittet, D. C. B. 1989, "The composition of dust in stellar ejecta," in Allanmandola, L. J., \& Tielens, A. G. G. M. (eds), Interstellar Dust, (Dordrecht:Kluwer), pp 455-466

Whittet, D. C. B. 2003, Dust in the Galactic Environment (2d ed.; Bristol: IoP)

Wilson, T. L., Mauersberger, R., Muders, D., Przewodnik, A., \& Olano, C. A. 1993, A\&A, 280,221

Witt, A. N., \& Gordon K. D. 1996, ApJ, 463, 681

Wolf, S., Henning, Th., \& Stecklum, B. 1999, A\&A, 349, 839

Xu, J., Crotts, A. P. S., \& Kunkel, W. E. 1995, ApJ, 451, 806

Wood, K., Haffner, L. M., Reynolds, R. J., Mathis, J. S., \& Madsen, G. 2005, ApJ, 633, 295

Young, P. A., et al. 2006, ApJ, 640, 891 
Table 1. Silicate dust heating/cooling

\begin{tabular}{ccc}
\hline \hline $\begin{array}{c}\text { Grain Size } \\
(\AA)\end{array}$ & $\begin{array}{c}\text { Heating Time } \\
(\mathrm{sec})\end{array}$ & $\begin{array}{c}\text { Cooling Time } \\
(\mathrm{sec})\end{array}$ \\
\hline 10 & 381 & 12 \\
20 & 50 & 125 \\
30 & 14 & 435 \\
40 & 6 & 1041 \\
\hline
\end{tabular}






Fig. 1.- Geometry of the IR light echo propagation. The red star represents Cas A. Assuming a dust column behind Cas A, the light-red colored region in each parabola (a two-dimensional parabolic surface parallel to the line of sight) defines the dust heated by light from the initial outburst at a specific time, $t_{1}<t_{2}<t_{3}$, since the supernova outburst. This heated dust produces the IR echoes. 


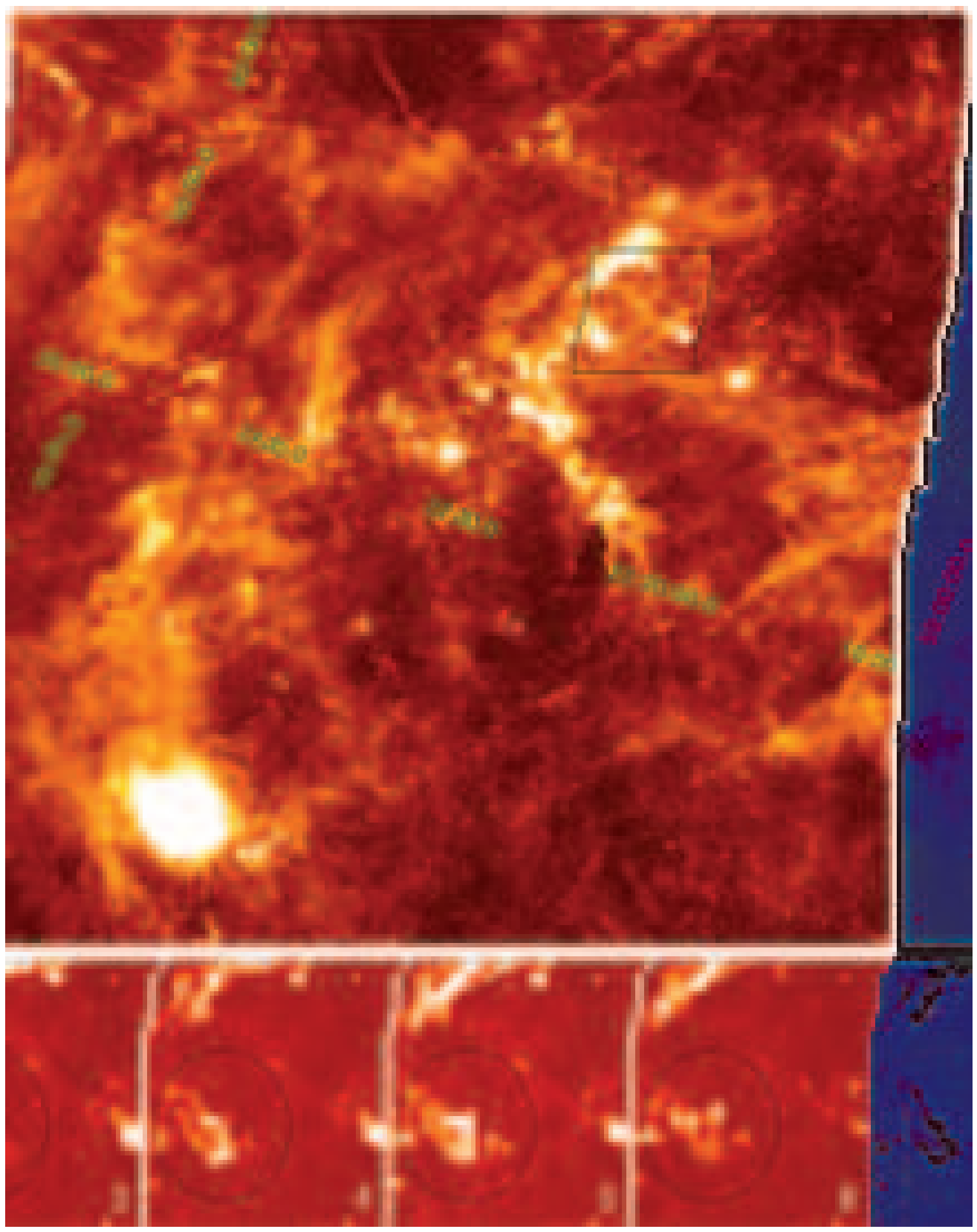

Fig. 2.- $24 \mu \mathrm{m}$ images of moving features (at the bottom) about $45 \mathrm{pc}$ in projected distance from Cas $\mathrm{A}$ in the northern region (the subregion shown in a square box). Cas $\mathrm{A}$ is the big bright blob on the left lower side of the top image. The images were obtained on 2005 February 3 (epoch 3), 2005 September 1 (epoch 4), 2006 February 14 (epoch 5), and 2006 October 2 (epoch 6). Nearly all the bright features in this region are IR light echoes, and they are especially evident in the circles. 

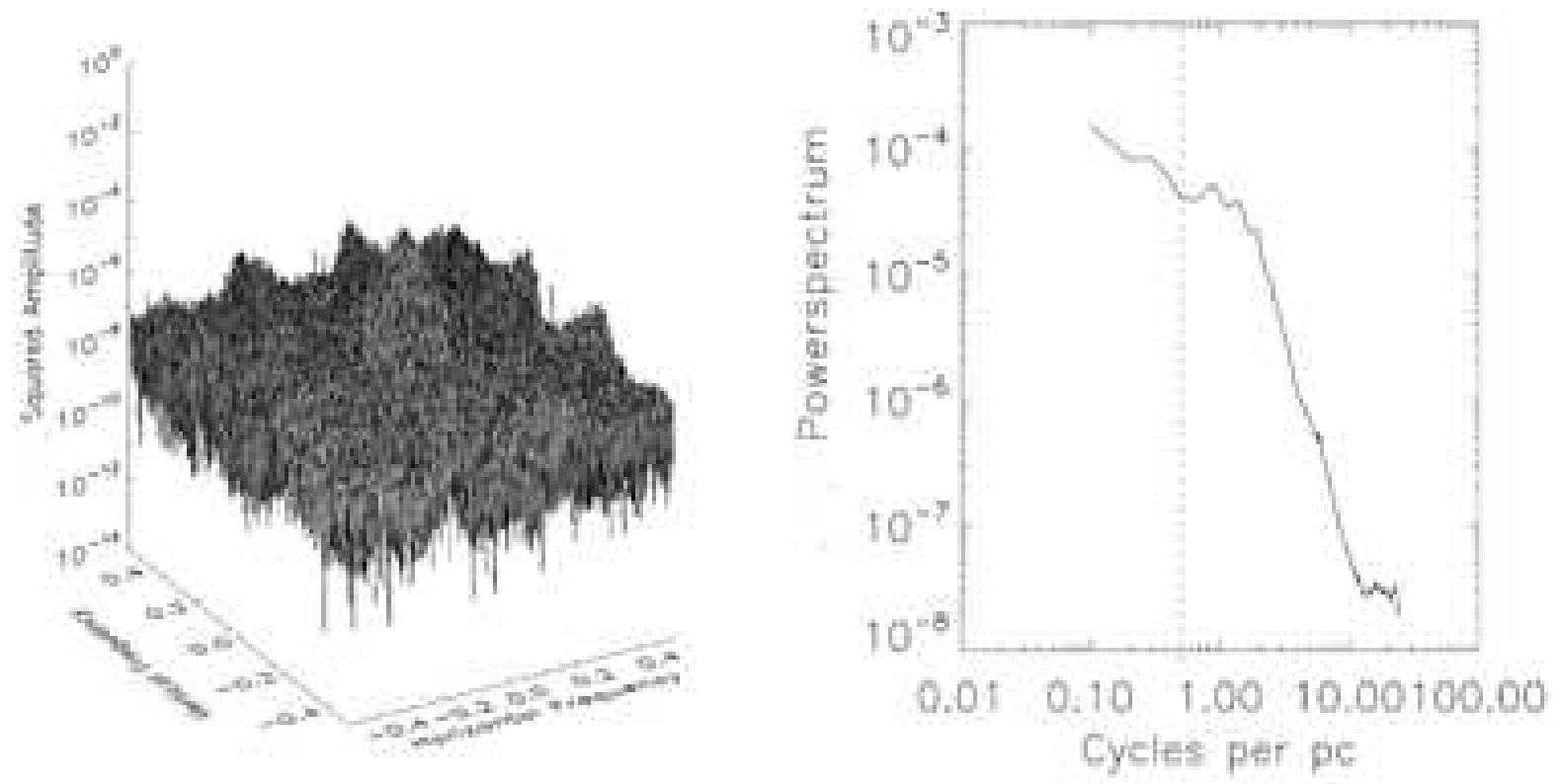

Fig. 3.- Surface plot of the power spectrum of the difference images (mostly IR light echoes, Cas A, bright stars, and nebulae) on the left and the averaged power spectrum in log scale on the right. The cutoff frequency, where the filter is half its maximum, is defined by the vertical dashed line. 


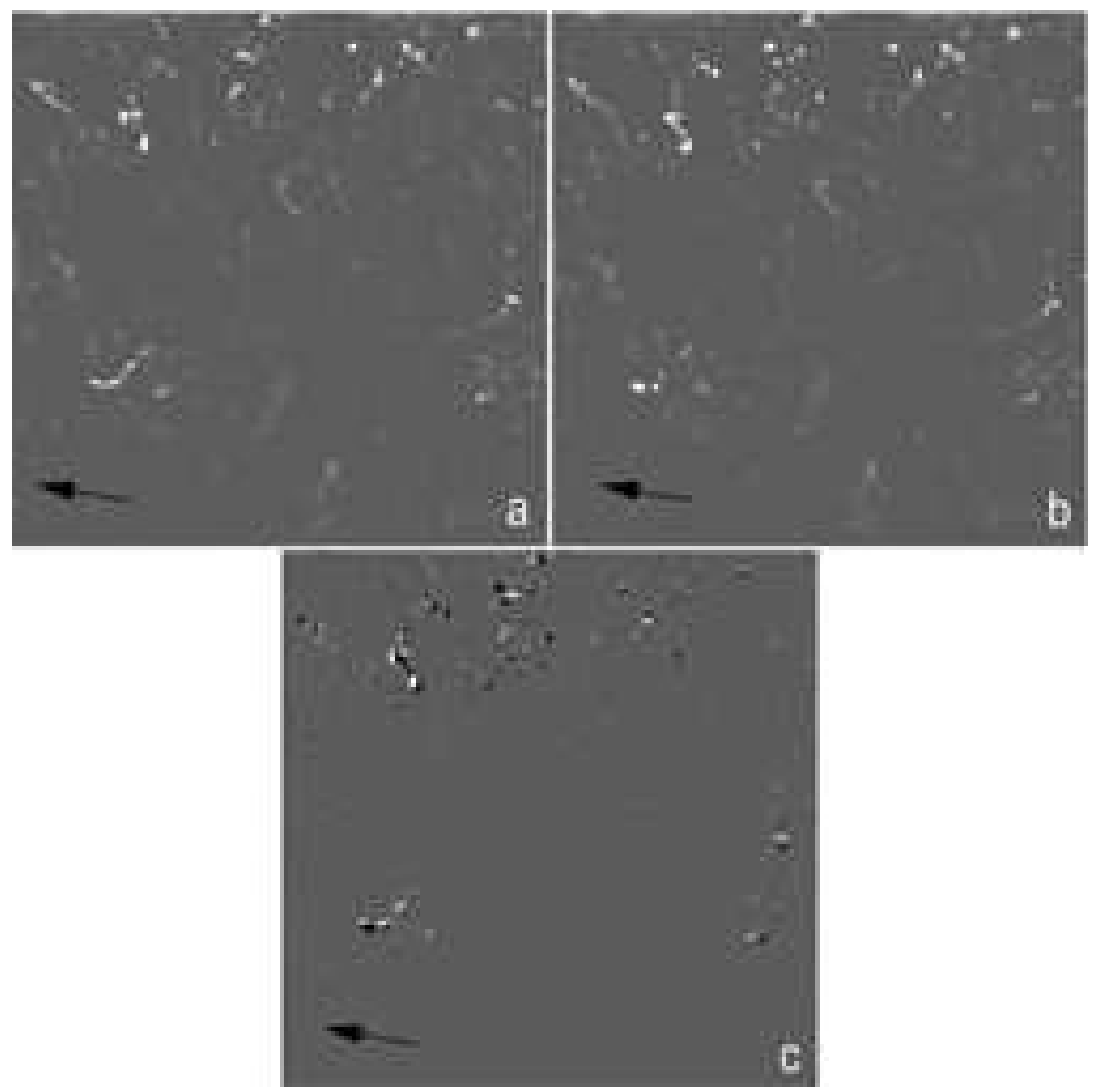

Fig. 4. - Subtraction of two different epoch images. Image (a) is a small region extracted from the epoch 4 image, while image (b) is from the epoch 5 image. The difference image is shown in (c). The bright white regions belong to epoch 4 while the black regions belong to epoch 5. The location of Cas A with respect to the subregions is indicated by arrows. 


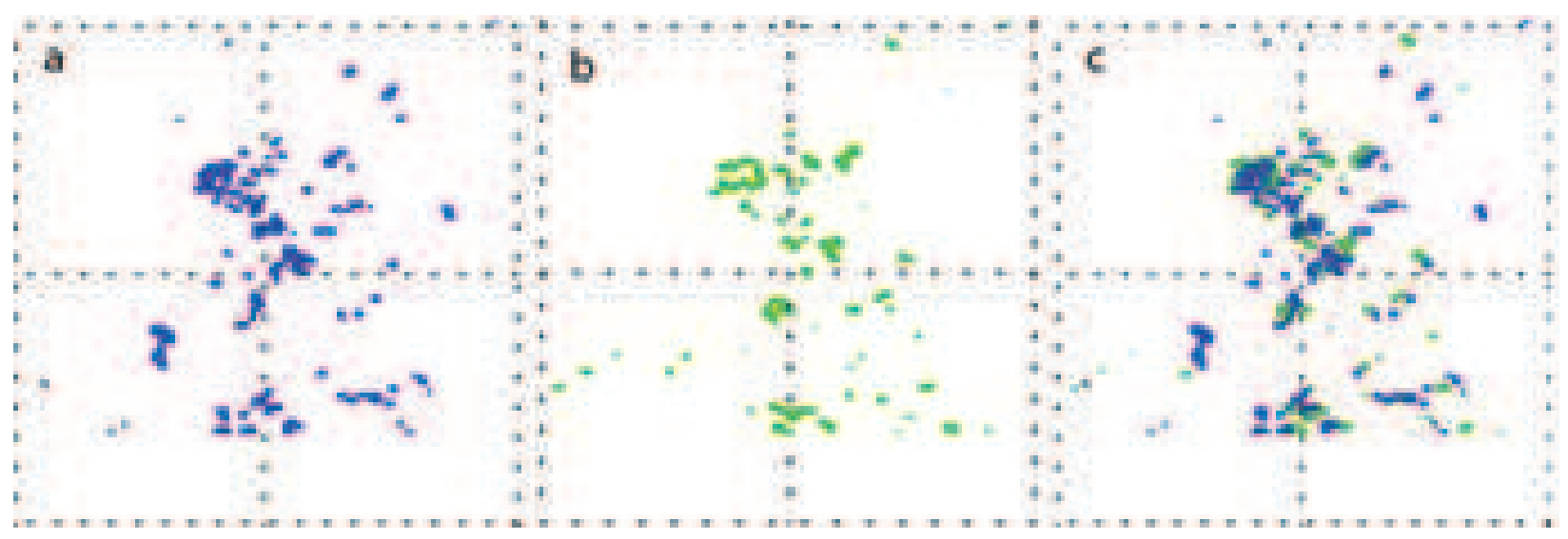

Fig. 5.- Detected pattern of ISM material for a $20 \mathrm{pc} \times 20 \mathrm{pc}$ region: a) is epoch 1 ; b) is epoch 2; and c) is their combination. 


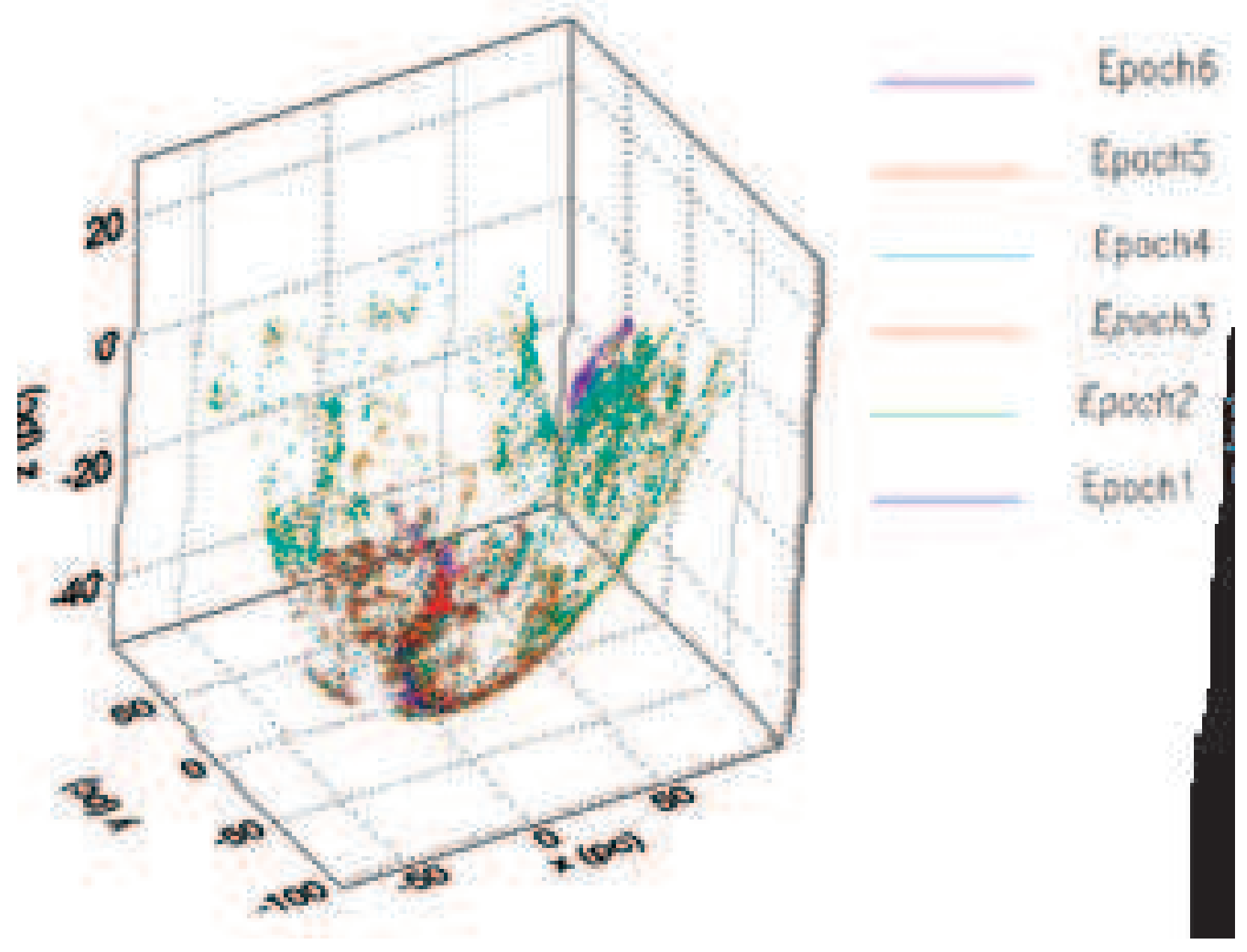

Fig. 6.- A three-dimensional plot of dust from all six epochs over about three years. Cas $\mathrm{A}$ is located at the origin $(0,0,0)$, and $z$ values increase toward the observer on Earth. The unit in $x, y$, and $z$ is parsecs. 


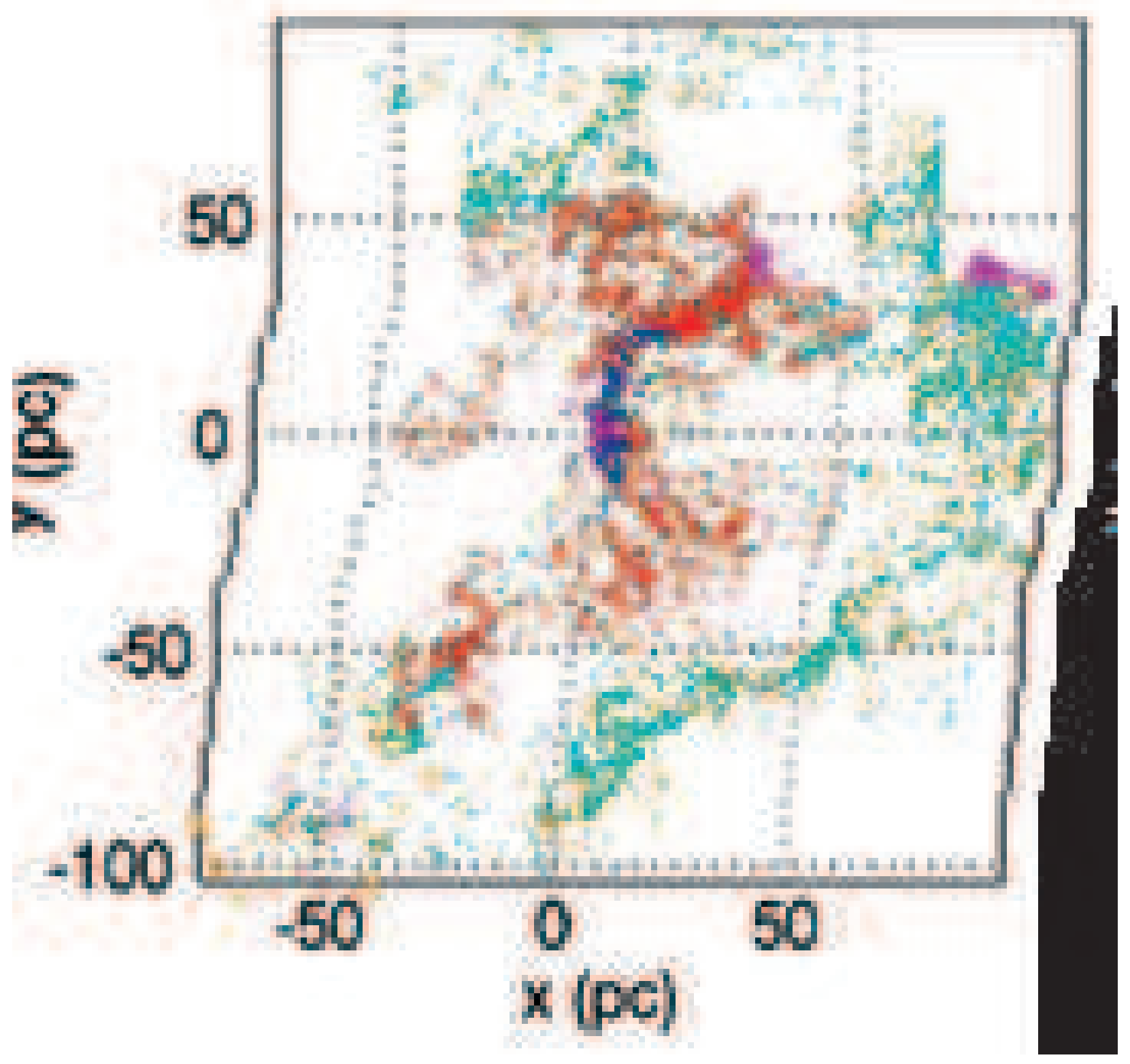

Fig. 7.- Projected image of the three-dimensional plot as viewed by a distant observer. Cas A is located at the origin $(0,0)$. The same color representations as in Fig. 6 are used. 

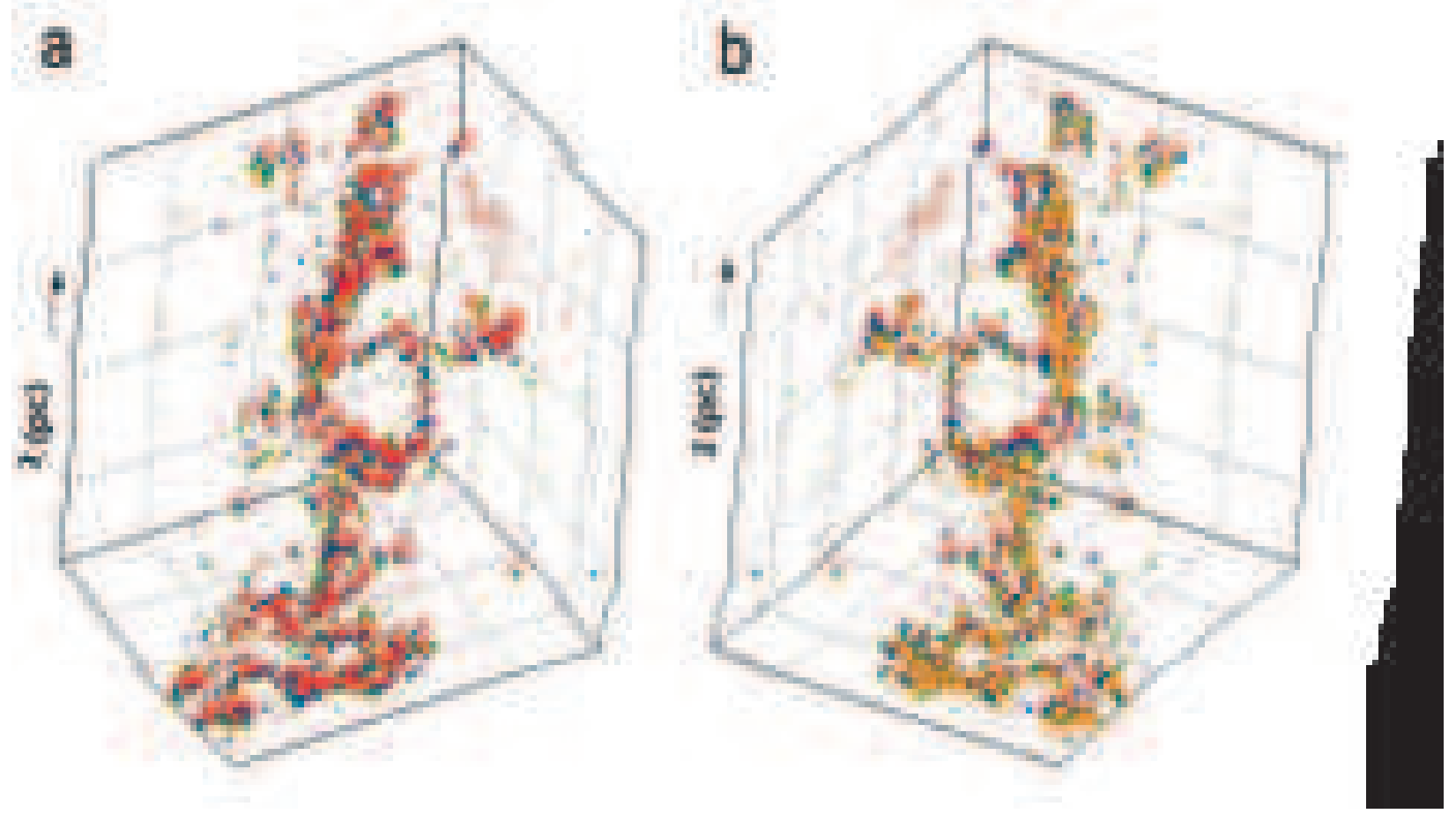

Fig. 8. - The two images show the three-dimensional structure of the ISM dust in the northern region of Cas A, from both sides. The dotted grid indicates a spacing of $2 \mathrm{pc}$ in the $\mathrm{z}$ axis (toward the observer) and $5 \mathrm{pc}$ in the $\mathrm{x}$ and $\mathrm{y}$ axes. The colors are red in epoch 3 , blue in epoch 4 , green in epoch 5 , and orange in epoch 6 . 




Fig. 9.- Filling factors in percent as a function of projected distance, $h$, in four different angular quadrants in epoch 4 . The dashed line represents the average filling factor, $0.4 \%$. 

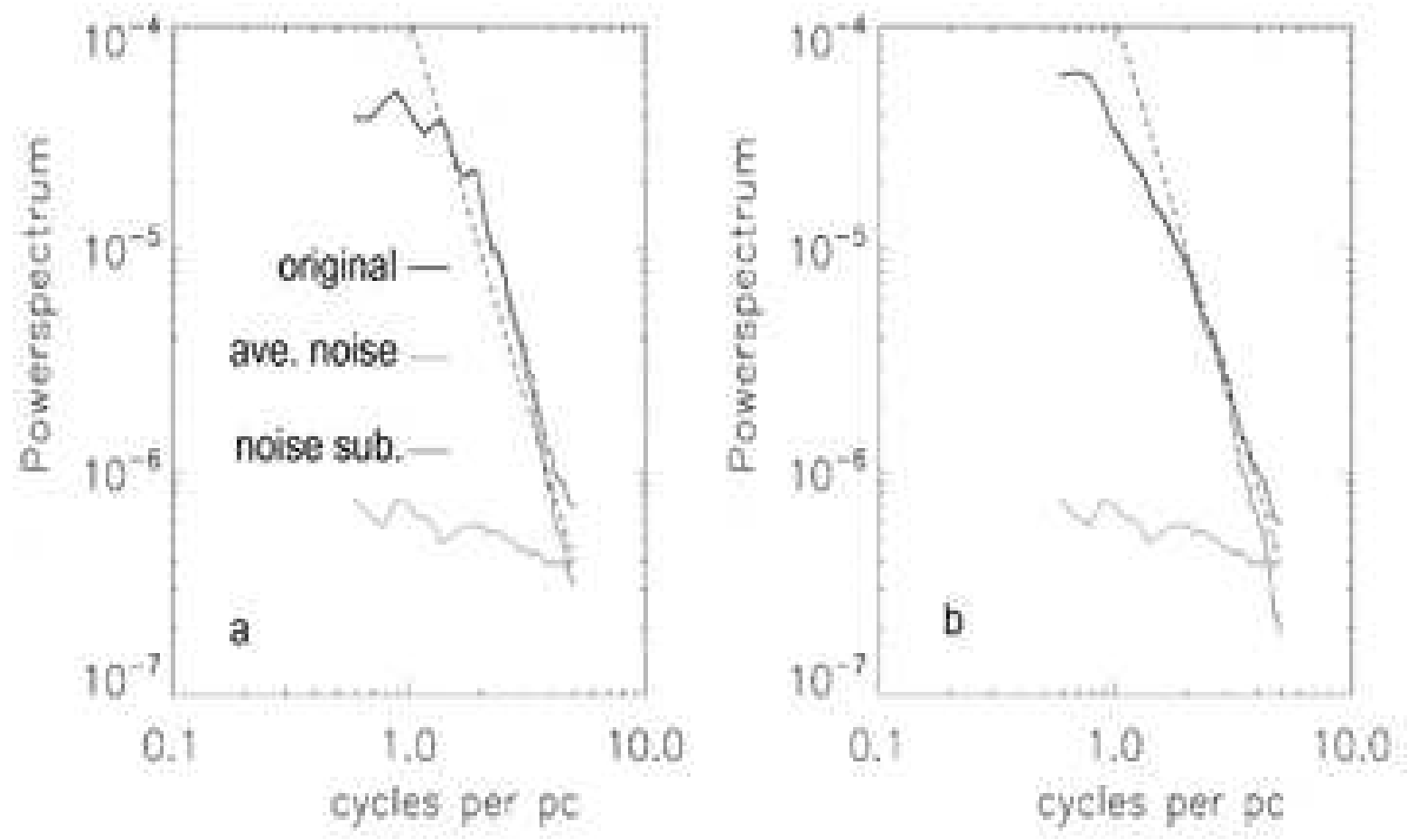

Fig. 10.- Power spectra of two sample regions. The lines are coded for both images as indicated in (a): original data (black solid line), the noise (red solid line), and noise-subtracted data (blue solid line). The dashed line represents a power law with a spectral index of 3.5. 